\title{
Eurostudia
}

\section{La « Nouvelle Hanse » de Björn Engholm a-t-elle vraiment été un échec?}

\section{Nicolas Escach}

Volume 7, numéro 1-2, 2011

Deutschland und Europa : Grenzen und Grenzgänge(r)

L'Allemagne et l'Europe : frontières et passeurs

URI : https://id.erudit.org/iderudit/1015012ar

DOI : https://doi.org/10.7202/1015012ar

Aller au sommaire du numéro

Éditeur(s)

Le Centre canadien d'études allemandes et européennes

ISSN

1718-8946 (numérique)

Découvrir la revue

Citer cet article

Escach, N. (2011). La " Nouvelle Hanse » de Björn Engholm a-t-elle vraiment été un échec ? Eurostudia, 7(1-2), 73-86. https://doi.org/10.7202/1015012ar

Tous droits réservés $@$ Le Centre canadien d'études allemandes et européennes, 2012
Ce document est protégé par la loi sur le droit d'auteur. L'utilisation des services d’Érudit (y compris la reproduction) est assujettie à sa politique d'utilisation que vous pouvez consulter en ligne.

https://apropos.erudit.org/fr/usagers/politique-dutilisation/ 


\begin{abstract}
Nicolas Escach
Centre interdisciplinaire d'études et de recherches sur l'Allemagne, École normale supérieure de Lyon
\end{abstract}

L

es années 1980 et 1990 ont été des années d'intense maturation en matière de reconstruction d'une unité baltique tant par les chercheurs que par les politiques. Alors que de nombreuses villes des espaces riverains s'étaient associées dans le cadre de jumelages dès 1975 et que la "Convention d'Helsinki pour la protection de la mer Baltique » avait été signée dès 1974, Björn Engholm, président du Land Schleswig-Holstein, futur président du SPD et futur candidat au poste de chancelier lance l'idée à Kiel en 1988 d'une "Nouvelle Hanse », faisant ainsi référence à une période historique glorieuse au cours de laquelle les comptoirs de la Baltique avaient activement coopéré pour sécuriser les approvisionnements maritimes et lutter contre la piraterie. Un retour du mythe de la Hanse aurait, selon lui, après plusieurs années de division au sein de l'Europe, une visée mobilisatrice tant les mémoires hanséatiques pourraient constituer une part indispensable à l'accomplissement des projets européens. Les villes de la Baltique semblent donc avoir redécouvert, en partie grâce à lui, depuis une trentaine d'années, l'héritage matériel et immatériel de la Hanse du Moyen Âge, qu'elles ont tenté d'ériger en ressource afin de fonder des réseaux économiques, politiques, institutionnels. Ces réseaux de villes, au cœur d'une gouvernance européenne de plus en plus multiscalaire et multi-niveau, sont une manière de dépasser les frontières étatiques et les ferveurs nationalistes, constituant des espaces de coopération transnationaux fondés sur une prétendue identité hanséatique régionale.

Pourtant, malgré ces nombreux apports, le personnage et son projet ont été, depuis son retrait politique en 1994, fortement critiqués à tel point que Rainer Burchart et Werner Knobbe, deux journalistes allemands, ont choisi comme titre pour leur biographie de Björn Engholm, Récit d'un espoir déçu. Accusé de nationalisme et de réactivation d'idées nazies, lâché par son camp politique le Ministre-Président a dû répondre de son action politique. La "Nouvelle Hanse», critiquée par plusieurs gouvernements et intellectuels des espaces riverains de la Baltique a-t-elle rassemblée ou divisée la région dans son ensemble ? Au-delà du concept en lui-même, il semble que le parcours, les idées et les actions de Björn Engholm aient permis d'esquisser théoriquement l'émergence d'une région baltique qui s'est développée en pratique par la suite. 
Les définitions de la région et donc du processus de régionalisation peuvent varier $^{1}$ : nombre limité d'États liés par une relation géographique et par un degré d'interdépendance mutuelle pour le géopoliticien Joseph Nye, unité d'aspects géographiques, politiques, économiques ou culturels et/ou une identité régionale qui sont caractérisés par un certain degré d'homogénéité et/ou de fonctionnalité pour le professeur en sciences politiques de l'université de Iéna Helmut Hubel². Les années 1980 voient en effet le retour du concept de région permis par la fin d'un monde bipolaire. Or ces régions sont indéniablement construites par les politiques. Joseph Nye affirmait que les régions sont « ce que les politiciens et le peuple veulent qu'elles soient ${ }^{3}$. Marko Lehti, chercheur finlandais, va dans ce sens lorsqu'il définit la construction régionale comme un processus politique où images et truismes sont construits politiquement ${ }^{4}$. Iver B. Neumann, spécialiste des relations internationales et géopoliticien ayant travaillé à l'Institut des affaires internationales de Norvège, s'inscrit donc dans cette lignée: «Les qualités spécifiques individuelles comme des particularités culturelles, économiques, géographiques et ou des événements historiques sont choisis consciencieusement pour imaginer sur leur base une identité temporelle et spatiale autour de l'unité ou de la communauté $»^{5}$. Les régions sont définies par des actes de langage politiques (speech acts) qui les font exister. Ce qui est présenté par les politiques comme pertinent culturellement est plus important que les similitudes culturelles elles-mêmes. On comprend alors mieux l'ouvrage de Karl Schröder qui parlait de «Spacing Story » dans Im Raume, lesen wir die Zeit.

En parlant de la Baltique comme d'une région unie culturellement, économiquement et politiquement, Björn Engholm a rendu possible l'existence de cette unité. L'école de Kiel qu'il a créée, à travers la Denkfabrik6, véritable centre de planification stratégique de l'unification baltique, a rendu possible l'élaboration de pistes de réflexion qui ont mené plus tard à de réelles avancées dans le processus de régionalisation. Le rôle du Schleswig-Holstein et de Björn Engholm dans la construction d'une région baltique (region-building) est essentiel. Le Land fut qualifié régulièrement de « livreur d'idées » (Ideenlieferant). Parallèlement, Björn Engholm a offert une nouvelle définition de la région, plus vaste. Il existe plusieurs définitions de «l'espace baltique » ou de la région baltique : la mer Baltique elle-même, le bassin hydrographique de la Baltique et l'ensemble des espaces de ce bassin du golfe de Finlande jusqu'aux détroits Skagerak/Kattegatt, la région fonctionnelle formée par les

\footnotetext{
${ }^{1}$ Nathalie Blanc-Noël (dir.), La Baltique. Une nouvelle région en Europe (Paris : L'Harmattan, 2002).

2 Helmut Hubel et Stefan Gänzle, The Council of the Baltic Sea States (CBSS) as a Sub-Regional Organization for "soft security risk management" in the North-East of Europe, Report to the Presidency of CBSS, 18 May 2001 (Iéna: Friedrich-Schiller-Universität, 2001).

${ }^{3}$ Joseph S. Nye Jr. (dir.), International Regionalism Readings (Boston: Little Brown, 1968).

${ }^{4}$ Hiski Haukkala (dir.), Dynamic Aspects of the Northern Dimension (Turku: Turku University Press, 1999).

${ }^{5}$ Iver-B. Neumann, « A Region-Building Approach to Northern Europe », Review of International Studies 20 (1991): 59.

${ }^{6}$ Littéralement: « la fabrique à pensées ».
} 
échanges économiques et les investissements, la région humaine définie par les densités de population, la région institutionnelle définie par les projets INTERREG IV-B et l'approche macro-régionale et la région historique fondée à partir des routes des Varègues puis celle des Hanseaten et des comptoirs hanséatiques (pouvant aller jusqu'au Portugal). En réalité, la Baltique tout comme l'espace hanséatique sont des « espaces-valise » ou «espaces-nomade». «L'espace-nomade ou espace-valise apparait donc comme un espace que l'on peut tirer à soi et que l'on peut déformer à sa guise car ce dernier n'a jamais été précisément délimité et ne pourra probablement pas l'être. L'espace est fantasmé, imaginé, recrée, recadré par les acteurs dans la perspective de leurs propres stratégies ${ }^{7}$. La Baltique de Björn Engholm, comme toute région historique et culturelle, est à géométrie variable, sanguine et réticulaire, espace de la circulation et du flux. Elle pose autant de points d'interrogation qu'elle apporte de réponses toute faites. Ces éléments sont-ils suffisants pour réhabiliter l'œuvre de Björn Engholm ? La « Nouvelle Hanse » a-t-elle un avenir ?

\section{LA NOUVELle HANSE, UN PROJET POUR LA MER BALTIQUe?}

\section{Björn Engholm : la vision d'un « Hanseat » social-démocrate}

Björn Engholm adhère au parti social-démocrate d'Allemagne dès 1962. Il cumule ensuite sous cette étiquette des fonctions nationales (élu député du Bundestag en 1969, Secrétaire d'État parlementaire du Ministre fédéral de l'Éducation et des Sciences dès 1977, puis ministre dès 1981, Ministre de l'Agriculture en 1982, Président fédéral du SPD en 1991 puis candidat à la chancellerie) et régionales (élu Ministre-Président du Schleswig-Holstein de 1988 à 1993 après avoir été longtemps dans l'opposition).

Son intérêt pour la Baltique et les pays scandinaves est en adéquation avec une position traditionnelle des sociaux-démocrates allemands sur l'Europe et sur la politique étrangère allemande. Depuis la fin de la Seconde Guerre mondiale, il est vrai que l'Allemagne avait peu coopéré avec les pays baltiques. Les contacts les plus étroits avec les pays d'Europe du Nord s'étaient justement réduits à ceux tissés par Willy Brandt, de par son exil en Norvège et en Suède et de par ses origines nordallemandes ${ }^{8}$, alors que du côté nordique, les tendances à l'isolement de la Suède ou de la Finlande s'étaient multipliés. Or Willy Brandt est un objet de fascination pour Björn Engholm qui publie en 1992 un recueil de textes lui rendant hommage : "Sa patrie politique, la social-démocratie doit rester comme elle a toujours été : ouverte au monde, européenne, patriotique, réformatrice, de gauche et libre ${ }^{9}$. Les discours de Björn Engholm sont véritablement pétris par des références sociales-démocrates. Il insiste sur la formule « l'Allemagne est notre patrie, l'Europe notre futur », cite Paul-

\footnotetext{
${ }^{7}$ Nicolas Escach, Une nouvelle Hanse en Europe, Brême, Gdansk, Riga (Berlin: Trafo Verlag, 2010), 175.

${ }^{8}$ Leena-Kaarina Williams, Zur Konstruktion einer Region. Die Entstehung der Ostseekooperation zwischen 1988 und 1992 (Berlin: Berliner Wissenschafts-Verlag, 2007).

${ }^{9}$ Björn Engholm, Dank an Willy Brandt (Marburg: Schüren Verlag, 1992), 75.
} 
Henri Spaak, Helmut Schmidt, Bruno Kreisky et défend un travail collégial où la concertation prime sur la hiérarchie verticale.

La deuxième raison qui a poussé Björn Engholm à s'intéresser aux pays scandinaves et à l'espace baltique est à trouver dans ses origines. Il loue chez Brandt «l'homme de Lübeck », expression qui sera reprise pour le qualifier. Rainer Burchart et Werner Knobbe n'hésitent pas à lui attribuer le titre d'Hanseat. Résolument nordallemand, distant et distingué, humaniste, modeste à l'excès, fier des traditions hanséatiques et de l'architecture de la «Perle de la Baltique », l'homme évoque de manière récurrente sa patrie baltique. Il se définit lui-même comme un Nordeuropéen, aspirant au consensus, préférant la pratique à de longs discours, recherchant la justice et le juste milieu.

\section{Le constat d'une Baltique en marginalisation, la peur d'une Europe à deux vitesses...}

Björn Engholm mène dès son élection à la tête du Land du Schleswig-Holstein en 1988 d'intenses réflexions sur l'espace baltique. Il entrevoit le danger dans une future Europe réunifiée d'une accentuation du modèle centre/périphérie. Dans cette perspective, les centres seraient l'Isthme européen s'étalant de Londres à Milan, la Sunbelt européenne connectant Milan à Lyon et la région (éventuellement) «postsocialiste » associant Prague, Budapest, Dresde et Leipzig. Les régions du Nord, subissant la concurrence de celles du Sud de l'Europe, se trouveraient, dans ce schéma, marginalisées. Afin d'éviter une «périphérisation » des pays nordiques, il serait pour lui urgent d'établir une coopération étroite dans le but de rassembler le potentiel de la région: une forte productivité, une force d'innovation, un haut standard écologique, la concentration de $20 \%$ du commerce mondial et de 70 millions d'habitants. Cette étroite association des pays baltiques est d'autant plus essentielle qu'elle doit répondre à un certain nombre de défis futurs : rendre les états baltiques assez forts pour que les négociations futures d'entrée dans l'UE et pour que la formation d'un marché économique commun arrivent à leur terme, présenter les intérêts communs baltiques d'une seule voix, notamment auprès de l'UE, coordonner les liaisons et les flux de transports dans le Nord de l'Europe afin que la régionalisation passe par des mobilités accrues au sein des espaces riverains, proposer une impulsion économique en se basant sur la très forte culture entrepreneuriale du nord de l'Europe (visible notamment dans le grand nombre de PME qui sont autant de potentiels d'innovation), aider les petites et moyennes entreprises, encourager un développement écologique, mener une coopération culturelle intense afin que l'identité baltique trouve une assise populaire.

Parallèlement, l'Europe s'accorde sur les valeurs communes de démocratie, de liberté, de pluralisme, de justice indépendante, et sur la fin du parti unique, de la censure et de l'économie planifiée. Si les blocs de l'Ouest et de l'Est ne sont plus une forme d'organisation, de classement et de structuration, selon quels critères, à partir de quelles règles les rapports entre les États vont-ils s'organiser? C'est ce vide qu'occupe l'idée d'une régionalisation baltique. 
La coopération proposée par Björn Engholm pourrait s'appuyer sur une forme de caractère et $\mathrm{d}$ 'habitudes communs des populations baltiques : «Ce qui nous relie, ce sont bien les mêmes standards sociaux, un tempérament et un caractère commun, des conditions de vie communes liées au paysage, aux températures, au climat, à l'influence maritime, aux formes de peuplement. Nous sommes calmes, absolument pas brutaux, souvent têtus mais dignes de confiance. Nous avons un sentiment aigu de la justice sociale ${ }^{10}$. À côté de cette «nature nordique », les pays riverains partageraient aussi une histoire commune, l'histoire hanséatique, la mémoire de mille ans de coopération, mémoire au cœur de la pensée de Björn Engholm.

\section{Le retour du concept de "Nouvelle Hanse »}

La redécouverte de la Hanse en Europe débute le 31 Octobre 1978 lorsque la ville de Zwolle lance un appel à 57 villes hanséatiques dont Bergen et Londres. La ville précise dans son invitation que l'idée de Hanse est un élément important car elle rappelle l'époque d'une coopération économique qui allait au-delà des frontières nationales. Zwolle organise ainsi du 23 au 27 août 1980 les premières journées de la Hanse (Hanstage) que la presse évoque à l'image du Westfälische Rundschau et d'autres journaux qui titrent: «La Hanse vit à nouveau». Ces rencontres marqueront la naissance du réseau de villes « Hanse des Temps Nouveaux ».

Dès le milieu des années 1980, certains élus du Schleswig-Holstein et politiques allemands promeuvent des collaborations économiques plus étroites entre le Land et les pays européens tels le Danemark et autres pays baltiques. C'est le cas d'Egon Bahr, originaire de l'Est, et du ministre du Schleswig-Holstein pour les affaires sociales, la santé et l'énergie, Günter Jansen. Egon Bahr milite dès les années 1970 pour des liens approfondis avec la Scandinavie et pour l'inclusion des pays de l'Est dans une "Hanse moderne». A côté des intérêts économiques, cette initiative présente également un objectif de réconciliation et de pacification. Cependant, les soutiens politiques restent peu nombreux tout comme l'attention portée au concept ${ }^{11}$.

Björn Engholm a une certaine vision de la Hanse. Pour lui, la Hanse représente une forme d'unité économique ancienne, une organisation flexible, sans date de création ni « contrat », qui a favorisé une forme de stabilité et de paix en Europe. La Ligue hanséatique serait synonyme de libertés intérieures, d'uniformisation des règles de commerce et $\mathrm{du}$ droit autour de la Baltique, de division internationale $\mathrm{du}$ travail et d'apparition d'un marché global d'échanges sans frontières ni barrières, qui aurait rapproché les hommes et les cultures. Sa vision de la Hanse n'est pas seulement spatiale mais reflète véritablement une " communauté de valeurs essentielles ». Le concept de "Nouvelle Hanse », utilisé à de multiples reprises au cours du XXe siècle, doit exprimer l'urgence d'une coopération et être directement mobilisateur : «La chance de coopérer est plus grande qu'auparavant, c'est la configuration la plus

\footnotetext{
10 « Die Neue Hanse », NordEuropaForum 04 (1991): 10.

${ }^{11}$ Williams, Zur Konstruktion einer Region.
} 
idéale depuis le début de la Guerre de Trente ans ${ }^{12}$. Björn Engholm donne de la Hanse une définition spatiale large, l'étendant jusqu'à Londres à l'Ouest, jusqu'à Cologne au Sud, jusqu'à Novgorod à l'Est et jusqu'au Norden scandinave au Nord.

Le concept de «Nouvelle Hanse », tel qu'il l'utilise, établit une véritable vision de la coopération baltique qui s'inscrit dans les prémices d'une «Europe des régions ». Le fait que l'idée vienne du Ministre-Président d'un Land allemand n'est pas étranger à une conception de la coopération supranationale, décentralisée et réticulaire. La région baltique n'est plus conçue comme un espace divisé par des frontières étatiques mais comme une entité historique, géopolitique réticulaire définie par la métaphore de la Hanse. Elle devient dans l'esprit de ses créateurs un réseau non hiérarchisé et supranational. Björn Engholm est très attaché à cette absence de hiérarchie : «Il ne s'agit pas de rappeler la domination germanique en Baltique. Bien au contraire, nous voudrions que le concept de Hanse soit associé à l'idée d'une coopération décentralisée, supranationale et régionale. Dans la future Europe des régions, ce ne seront pas les État-nations mais les unités régionales indépendantes qui coopéreront ensemble ${ }^{13}$. L'horizontal doit donc remplacer le vertical dans un ensemble qui s'oppose à une " société en pyramide ». Les acteurs non étatiques sont aussi présents que les acteurs étatiques.

De cette nouvelle vision de l'espace, découle une définition « postmoderne » de la région. Contrairement à la région moderne qui est territoriale, basée sur l'État, la région "postmoderne» est fondée sur le lien, les flux et le réseau. La question décisive de la région « postmoderne » est " être ou non relié ». L'État-nation aurait été une interprétation subjective et datée de la politique spatiale. Ces éléments fondent la différence entre l'ancien régionalisme ( Old Regionalism»), constitué d'organisations intergouvernementales, et de relations multilatérales et le nouveau régionalisme ( New Regionalism») que les intellectuels de la Denkfabrik entendent bâtir se concentrant sur la mise en réseau des acteurs des pays riverains, la soft security et une consultation de la société civile. Björn Engholm promeut la gouvernance et non le gouvernement, le bottom up plutôt que le top down, le processus plutôt que la structure, l'ouverture et non la fermeture, la coopération plutôt que la coordination, la confiance plutôt que la responsabilité, la décentralisation plutôt que la concentration ; en somme un modèle « néo-médiéval » et non « néo-westphalien ».

\section{LA « NOUVELLE HANSE », L'HISTOIRE D'UN ECHEC ?}

L'échec de la «Nouvelle Hanse » coïncide avec la chute de l'homme

Dès 1992-1993, Björn Engholm connaît des difficultés personnelles et entre dans une période de grave crise politique qui aboutira à son départ de la vie politique en 1994. Cette crise est d'abord celle de la social-démocratie qui l'a porté ; or de cette

\footnotetext{
12 Ibid., 9.

${ }^{13}$ Ibid., 10.
} 
social-démocratie, il est une forme d'incarnation. Les arguments politiques s'appuyant sur la récession mondiale, sur la fin de la guerre froide, sur une nouvelle politique à l'Est et sur l'instauration de la démocratie sont dépassés. Candidat à la chancellerie, Björn Engholm ne propose pas un véritable programme et la politique européenne du SPD, fer de lance de la vision social-démocrate, ne s'est pas adaptée à la réalité du traité de Maastricht et des processus monétaires et économiques engagés. Il finit par démissionner de son poste de Ministre-Président le 13 mai 1993 suite à «l'affaire Barschel » qui parachève sa chute. Il doit en effet en 1993 reconnaître avoir menti devant une commission d'enquête du Landtag à propos de l'espionnage électoral dont il avait été victime de la part du Ministre-Président Uwe Barschel lors des élections de 1987. En 1994, il démissionne de son mandat parlementaire régional et se retire de la vie politique.

\section{Une critique du concept de «Nouvelle Hanse » autour de la Baltique}

Dès 1994, le concept de « Nouvelle Hanse » n'est plus autant utilisé par les élus même si l'idée d'une coopération dans la région demeure forte. En Scandinavie, côté danois et norvégien, la Hanse est surtout perçue comme une période de domination et de tutelle allemande à ne pas reproduire. La Hanse de Björn Engholm parait idéalisée. Plus qu'une période de paix et d'unification, elle semble avoir profondément divisée la région. Dès 1397, les royaumes de Suède, du Danemark et de Norvège signent «l'Union de Kalmar » pour lutter contre la suprématie de la Hanse en baltique. La Hanse a mené de nombreuses guerres en Baltique comme celle contre Waldemar IV, roi du Danemark, aboutissant à la paix de Stralsund. Uffe EllemannJensen, ministre des affaires étrangères danois ne se prive pas de le rappeler en 1992 : « Nous avons, nous Danois, mené une guerre contre la Hanse. Il faut rappeler que la Hanse avait comme centre Lübeck, comme langue l'allemand, et que son influence s'étendait de Novgorod à Londres ${ }^{14}$.

L'idée de «Nouvelle Hanse » apparait donc ambiguë. Cette interprétation est renforcée par deux éléments : certains propos tenus par Björn Engholm lui-même et le passé historique du concept de « Nouvelle Hanse ». Lors d'un discours à Brême le 29 mai 1991, Björn Engholm a la maladresse de situer l'Allemagne au centre du processus baltique: "Je n'évoque pas une Europe faible avec en son centre une Allemagne devenue plus grande mais une Europe forte dans laquelle l'Allemagne devenue plus grande injecte toute sa force ${ }^{15}$. Cette rhétorique nationaliste n'est pas sans rappeler un autre passé.

Depuis la chute de la Hanse historique à l'époque moderne, et particulièrement dès le XIXe siècle, le mythe hanséatique est réapparu à de nombreuses reprises souvent sous forme d'une manipulation politique ou idéologique. Il s'agissait alors

${ }_{14}$ Rainer Burchart et Werner Knobbe, Björn Engholm: Die Geschichte einer gescheiterten Hoffnung (Stuttgart: Deutsche Verlags-Anstalt, 1993), 209.

${ }^{15}$ Alfred Gertler, Björn Engholm im Gespräch (Berlin: Bouvier Verlag, 1991), 209. 
d'évoquer une unité culturelle fictive au sein de la Baltique, alors même que les pays qui la composaient étaient de nature différente. Jusqu'à la Seconde Guerre mondiale, cette unité baltique a plutôt servi les intérêts germaniques. Alfred Rosenberg, futur «Ministre du Reich pour les territoires occupés de l'Est» vit dans la Hanse une manière de démontrer, nature à l'appui, la légitimité des Allemands à occuper la région. Hinrich Lohse, haut dignitaire nazi, membre de la Commission pour les territoires occupés à l'Est, déclara ainsi en 1942: «Les fluctuations de l'histoire reviennent comme un reflet et témoignent toujours de la domination allemande (...) notre Baltique est redevenue une mer allemande ${ }^{16}$. La Hanse vint en appui au concept hitlérien d'espace vital (Lebensraum) constituant une période de forte expansion allemande. L'entre-deux-guerres fut marqué, quant à lui, par les œuvres de l'historien Fritz Rörig. Celui-ci déclare en 1937: «Ce qui était appelé "ville" dans la Baltique restait immature et la plupart du temps primitif. Lübeck était mature ${ }^{17}$. Il évoqua l'unification de la Baltique sous les traits allemands : «Ce n'est qu'après 1250, c'est-à-dire après que les villes allemandes eurent été fondées, que l'on put parler de région baltique (Ostseeraum) dans le sens d'une unité économiquement pleinement organisée ${ }^{18}$. Fritz Rörig rapprochait l'apparition d'une région baltique de l'unification sous domination germanique. Il parlait de l'Allemagne comme d'une «porteuse de culture » et compta comme « quantité négligeable » la contribution des régions du Nord et de l’Est de l'Europe.

Une coopération trop «économique » une «éco-Hanse»?

Une critique inattendue de la « Nouvelle Hanse » se cristallise également autour de la candidature verte aux élections régionales du Schleswig-Holstein de 1992. Pour eux, le concept de «Nouvelle Hanse» prenant comme ancrage symbolique une période de prospérité économique s'accompagne d'une vision de la croissance baltique anti-écologique: «Des slogans comme la "Nouvelle Hanse", la région baltique, la Mare Balticum sont souvent utilisés. Les gouvernements et acteurs privés de Kiel à Stockholm et de Copenhague à Riga voient la région baltique comme une région de croissance économique au sein de l'Europe. Il est extrêmement contestable que cette vision de la croissance soit capable de résoudre les problèmes écologiques de la région. Il y a des raisons de penser qu'une expansion de la croissance en Baltique entrainera des dommages environnementaux supplémentaires et une détresse sociale ${ }^{19}$. Cette revendication des écologistes allemands a donné lieu à une conférence intitulée «Des observations vertes pour la région baltique » organisée à

\footnotetext{
16 Thomas Hill, «Die Neue Hanse : Rückblick eines Historikers auf einen Mythos », in Mare Balticum 1996 (Lübeck: Ostsee Academie, 1996), 20.

17 Jörg Hackmann, « Not only Hansa. Images of History in the Baltic Sea Region », in Mare Balticum 1996 (Lübeck: Ostsee Academie, 1996), 27.

${ }^{18}$ Fritz Rörig, «Hanse, Ostseeraum und Skandinavien », in Völker und Meere, Aufsätze und Vorträge, dir. Egmont Zechlin et al. (Leipzig: O. Harrassowitz, 1944), 141.

${ }^{19}$ Williams, Zur Konstruktion einer Region, 217.
} 
Copenhague au moment même où un Conseil des États de la Baltique était créé, en 1992.

\section{REHABILITER LA « NOUVELLE HANSE », EST-CE POSSIBLE ?}

La « Nouvelle Hanse » : le projet d'un Land

La « Nouvelle Hanse » a d'abord eu un effet concret à l'échelle locale. Grâce à Björn Engholm, le Schleswig-Holstein est le premier de deux Länder allemand à voir dans la région baltique une ressource possible. De 1988 à 1992, en développant le concept de "Nouvelle Hanse » à l'échelle subrégionale, il critique fortement la politique du gouvernement CDU qui l'avait précédé et qui s'était plutôt concentré sur les partenariats avec l'Asie autour des marchés high-tech et des nouvelles technologies (Chine, Corée, Japon, Indonésie). La proximité géographique des pays de la Baltique avec le Land est un argument essentiel pour une réorientation : «Les contacts avec les pays scandinaves sont en retard depuis plusieurs années. La Scandinavie est pour nous le partenaire le plus proche. Je n'ai rien contre les relations avec la Chine mais Copenhague se situe plus près de nous que Pékin, c'est pourquoi nous voulons faire le premier pas $»^{20}$. De son côté, l'opposition CDU insiste sur le caractère rétrograde d'une référence quelconque à la Hanse, morte depuis l'époque de la découverte de l'Amérique. L'avenir du Schleswig-Holstein se situerait plutôt en Europe de l'Ouest ou dans les zones pacifiques ou est-asiatiques.

Björn Engholm a une ambition pour son Land : le voir un jour devenir une plaque tournante des échanges maritimes et terrestres en mer Baltique. Il cherche donc à replacer le Schleswig-Holstein au centre de la région. Dans un contexte de réunification, il compte parmi les plus pauvres Länder d'Allemagne et a intérêt à construire des intérêts en mer baltique indépendamment de Bonn ou Berlin ${ }^{21}$. La structure fédérale de l'Allemagne est alors un atout pour cette construction. Le concept de « Nouvelle Hanse » devient synonyme de coopération autour de la Baltique renforcée avec le Schleswig Holstein comme pays-pont entre les régions de la Baltique, et de l'Europe de l'Ouest et du Centre. L'objectif est notamment de créer un axe Allemagne du Nord / Baltique qui viendrait contrecarrer l'axe Nord-Sud, passant par Hambourg, lequel évite les Länder du Schleswig-Holstein et du MecklembourgPoméranie-Occidentale.

Dans cette volonté de transformer le Land en plaque tournante, rien n'est négligé. Le vocabulaire utilisé est sans appel : «porte », " espace de transit » (Umschlagplatz), « plaque tournante», « hub», « centre», « espace du Norden», « région de l'Europe des régions ». Ces métaphores traduisent le besoin d'un discours du centre voire de la centralisation et même du centralisme qui doivent faire oublier l'effective situation

\footnotetext{
${ }^{20}$ Ibid., 62.

${ }^{21}$ Reinhold Wulff et Manfred Kerner, «Die Neue Hanse, » Arbeitspapiere des Instituts für Internationale Politik und Regionalstudien 1 (Institut für Internationale Politik und Regionalstudien des Fachbereichs Politische Wissenschaft der Freien Universität Berlin, 1994).
} 
de périphérie et de marginalisation que connait le Land. La géographie européenne est recomposée pour mettre en avant un atout de localisation du Land: il ne se situerait pas en marge mais au cœur du Norden, possédant des ouvertures vers la Scandinavie et pays de l'Est. Il pourrait être un guide montrant la voie de l'Est au Danemark, à la Norvège, à la Suède ou à la Finlande.

\section{Björn Engholm : le moteur de l'insertion allemande en Baltique?}

L'Allemagne réunifiée s'est montrée hésitante dans ses relations extérieures avec la Baltique. Quatre raisons peuvent expliquer ce changement de position de l'Allemagne (par l'intermédiaire du ministère des affaires étrangères) dans la région baltique. Tout d'abord, l'Allemagne a longtemps refusé de prendre position dans la démarche entreprise par les États baltes afin d'intégrer l'OTAN et l'UE ainsi que sur le contentieux de Kaliningrad pour ménager ses relations avec la Russie. En réalité, la République fédérale a été dans un premier temps favorable à la reconnaissance de l'indépendance des États baltes et fut même parmi les premiers pays à la proclamer en 1991 par l'intermédiaire de Hans Dietrich Genscher, connu pour son engagement envers l'Est de 1'Europe ${ }^{22}$. L'Allemagne, qualifiée alors d'avocat des Baltes (Anwalt der Balten) soutient la candidature des pays baltes dans la Conférence sur la sécurité et la coopération en Europe (CSCE), le Conseil européen, le Conseil de coopération de l'OTAN, le Conseil des États de la mer Baltique. Cependant, cet engagement décroît avec le départ de Hans Dietrich Genscher du ministère des affaires étrangères (19821992) et l'arrivée de Klaus Kinkel (1992-1998). Pour ménager la Russie, l'Allemagne soutient alors les candidatures de la Pologne, de la République Tchèque et de la Hongrie mais pas celle des Pays baltes. Une deuxième raison au retrait allemand est à trouver dans la perception allemande du partage des partenariats européens ${ }^{23}$. La République fédérale aurait reçu la responsabilité d'un parrainage des pays de l'Europe médiane et orientale comme la République tchèque ou la Hongrie alors que les relations avec les pays baltiques incomberaient aux pays nordiques (Norvège, Suède, Finlande) à travers les outils de coopération nordiques ou nordico-baltiques. Une troisième raison à cette indifférence est lisible dans la géographie de la Baltique elle-même. D'un côté, la Baltique est longtemps restée un marché étroit de seulement 50 à 60 millions d'habitants et d'un autre côté, l'Allemagne comme la Russie ou la Pologne ne possède qu'une petite partie de son territoire en Baltique (Hambourg, le Schleswig-Holstein et le Mecklembourg-Poméranie Occidentale ne représentent que $11 \%$ de la superficie allemande et moins de $8 \%$ des habitants) et est partagée entre mer Baltique et mer du Nord (où se situent la plupart des grands ports allemands). Enfin, un dernier handicap de l'espace baltique notamment depuis 2004 est que celui-

22 Helge Dauchert, « Deutschlands Baltikumpolitik, zwischen europäischer Integration und Annäherung an Russland », Nordeuropa Forum 2/2008: 54-73.

${ }^{23}$ Nikolaus Werz, Jenny Bonin, Jeanette Edler, Ute Fabricius, Christoph Krüger et Heribert Saldik, «Kooperation im Ostseeraum, Eine Bestandsaufnahme der wissenschaftlichen und politischen Kooperation unter besonderer Berücksichtigung der neue Bundesländer », Rostocker Informationen zu Politik und Verwaltung 24 (2005). 
ci recoupe peu ou prou l'espace européen. L'Allemagne a ainsi longtemps cru qu'il suffisait de s'investir dans les institutions européennes pour finalement $s^{\prime}$ investir dans la zone baltique elle-même. Même les rapports avec la Russie pourraient être réglés par la politique de voisinage de l'UE. Même lorsque les intérêts allemands en Baltique ont été reconnus, la politique menée dans la région n'a pas été visible: Gerhard Schröder a ainsi déclaré que la Baltique représentait une part aussi importante de la politique extérieure allemande que les USA et le Japon mais il était toujours question dans les médias du Japon et des USA et peu de la Baltique.

Ce relatif détachement envers la Baltique est spécifique à l'Allemagne si l'on compare l'ensemble des pays riverains et a longtemps mis les Länder du Nord de l'Allemagne en porte-à-faux par rapport à l'échelle fédérale comme le précise Nikolaus Werz : «Il existe une asymétrie dans l'intérêt porté par les pays riverains à l'espace baltique. L'Allemagne qui borde la Baltique grâce à seulement deux Bundesländer n'y voit pas un centre économique ce qui freine les efforts entrepris par le Mecklembourg-Poméranie-Occidentale, le Schleswig-Holstein et Hambourg pour porter leurs intérêts dans la région $»^{24}$. Cette situation a d'autant plus gêné les Länder du Nord que l'Allemagne fédérale est en principe responsable juridiquement de la politique extérieure. Le paragraphe 3 de l'article 32 de la constitution allemande mentionne la possibilité pour les Länder, avec l'approbation du gouvernement fédéral et dans la mesure de leur compétence législative, de conclure des traités avec des États étrangers. Ce paragraphe de l'article 32 a donné lieu à une variété d'interprétations allant du parallélisme affirmé entre Bund et Länder à l'affirmation d'une suprématie absolue de l'échelle fédérale. L'accord de Lindau, conclu en 1957, précise ce point en prévoyant que lorsque la fédération conclut des traités qui, de l'avis des Länder, touchent à leurs compétences législatives exclusives, le consentement des Länder est nécessaire avant que le traité ne devienne obligatoire en droit international. La constitutionnalité de l'accord n'est cependant pas reconnue de manière unanime. A l'échelle européenne, les transformations de la loi fédérale en 1992 et notamment le texte de l'article 23 renforcent ce flou juridique.

Puisque la RFA fut longtemps absente des processus de régionalisation baltique, les Länder ont dû se substituer à l'échelon fédéral pour défendre leurs intérêts dans la région. Ils se sont un temps comportés comme des États à part entière. Le SchleswigHolstein, premier des deux Länder à avoir entamé une politique offensive dirigée vers la Baltique, par l'intermédiaire de son Ministre-Président, Björn Engholm, a en effet souvent mené une politique étrangère indépendante ${ }^{25}$. Si l'idée d'un "Conseil des États de la mer Baltique » provient tout d'abord en 1990 de Mats Hellström, ministre suédois, auteur d'un "Plaidoyer pour un Conseil des États de la mer Baltique", celle-ci a été développée et exploitée en coopération avec Björn Engholm. En avril 1991, Björn Engholm, Werner Jann et Bernd Rohwer de la Denkfabrik se rencontrent à

\footnotetext{
${ }^{24}$ Ibid., 160.

${ }^{25}$ Williams, Zur Konstruktion einer Region, 57.
} 
Kiel. Le Ministre-Président du Schleswig-Holstein accuse directement l'État fédéral : «Cela est, pour notre part, d'une importance toute particulière car à l'échelle nationale, il n'y a ni intérêt, ni compréhension pour l'urgence de la coopération régionale ${ }^{26}$. Il enchaîne les déplacements officiels : à la Commission européenne pour rencontrer Jacques Delors ou à Moscou où il participe, en septembre 1991, avec Pierre Mauroy, Pertti Paasio et Alfonso Guerra, Mikhail Gorbatchev, Boris Eltsine et Eduard Schewardnadse, à la mise sur pied d'un Conseil qui réunirait le Schleswig-Holstein (et non le Bund), la Scandinavie, les Pays Baltes et la Russie. Björn Engholm se rend ensuite en Pologne afin de s'entretenir avec Lech Wałesa et son ministre des Affaires étrangères. Un voyage dans les Pays Baltes est cependant interdit par l'État fédéral qui rappelle au Ministre-Président qu'il ne peut pas inviter les chefs de gouvernements d'États nationaux à une conférence des présidents et chefs de gouvernements sans son accord préalable. Dérogeant à cette règle, Engholm envoie des invitations, avant que les Affaires étrangères ne finissent par se rallier à l'idée d'un sommet. De nombreux débats opposent alors le ministre Genscher et Engholm sur la place que pourraient occuper les Länder dans un Conseil d'États de la mer Baltique. Genscher propose que la délégation allemande à ce Conseil comporte un représentant des Länder. Le premier Conseil des États de la mer Baltique a finalement lieu les 5 et 6 Mars 1992 à Copenhague.

Grâce à l'action de Björn Engholm, de nombreuses institutions baltiques ont été mises en place notamment en collaboration avec d'autres gouvernements sociodémocrates comme celui d'Ingvar Carlsson en Suède, vice-ministre d'Etat de 1982 à 1991. L'Ars Baltica, réseau culturel autour de la Baltique est imaginé en juillet 1989. Cette plate-forme doit permettre de rapprocher les cultures baltiques : "Il s'agit d'un pas important afin de montrer et de faire prendre conscience de la similitude des formes artistiques et culturelles dans le passé, présent et futur. Les participants voient en Ars Baltica un renforcement culturel de l'espace baltique» ${ }^{27}$. En collaboration avec Pehr Gyllenhammar, le président du Land créé également en septembre 1990 le Club Nord Européen (North European Club) à Stockholm. L'objectif du NEC est de renforcer politiquement et économiquement l'unité baltique et le rapprochement entre l'Allemagne, les pays scandinaves, les pays de l'Est et les Pays Bas. Les trois grands thèmes de débats sont: les infrastructures de transport, la protection de l'environnement et les infrastructures sociales. Les membres du Club sont des chefs d'entreprises influents: Ulf Sundquist, directeur de la banque STS à Helsinki ou Harald Norvik, directeur de Statoil à Stavanger. À compter de mars 1989, apparaissent les Forums du Schleswig-Holstein qui permettent une réflexion et une rencontre entre de nombreux acteurs baltiques. Le premier forum sur le thème Mare Balticum attire des délégations de Pologne, des pays scandinaves et de RDA autour de Paulina Mürl (SPD). Björn Engholm a enfin largement contribué à la création en 1991 à Helsinki de la Conférence des parlementaires de la mer Baltique (BSPC) et, nous

\footnotetext{
${ }^{26}$ Ibid., 171.

${ }^{27}$ Ibid., 146.
} 
l'avons vu, à celle du Conseil des États de la Baltique (Copenhague, 1992). Ce dernier reste un lieu privilégié d'échanges entre le Bund et les Länder sur la coopération baltique. Les Länder y sont représentés au sein de la délégation allemande par un Land différent chaque année (le Schleswig-Holstein, le Mecklembourg-PoméranieOccidentale, ou Hambourg).

\section{Une nouvelle vision de la coopération baltique}

Grâce à Björn Engholm, la régionalisation baltique a avancé dans trois directions principales. Tout d'abord, celui-ci a théorisé, comme d'autres avant lui, une forme d'unité baltique. Il a mis en avant les similitudes des régions et pays riverains, après de longues années de division. Ensuite, il a offert la possibilité à des élus, chefs d'entreprise et artistes de se rencontrer à travers des plateformes accélérant la prise de contact ; or ces contacts serviront plus tard à élaborer de nouveaux projets. Enfin, par la métaphore de la Hanse, il a défini d'une nouvelle manière la région baltique. Celle-ci adopte des limites floues mais extensibles. Les réflexions autour de la création du Club Nord Européen posent la question de l'intégration potentielle des Pays-Bas. Björn Engholm l'affirme sans détour : « Nous ne devons pas oublier que les Pays-Bas ont avec nous une conscience régionale commune ou qu'ils l'auront par cette institution ${ }^{28}$. Cette phrase montre bien que Björn Engholm a davantage cherché à élargir l'espace baltique par l'institution qu'à recouvrir une conscience commune préexistante par l'institutionnalisation. L'institution crée la région et non l'inverse. Le Conseil des états de la Baltique comprend quant à lui la Russie. Les réseaux hanséatiques se sont étendus au Moyen Âge de manière large en Belgique, Pays-Bas, Russie, Biélorussie, Angleterre. Björn Engholm ne fait pas de la Baltique une région figée : il a souvent l'habitude de la représenter sous le trait de cercles concentriques.

\section{CONCLUSION}

La coopération baltique avait pris son essor suite à la chute du rideau de fer mais connait une crise depuis quelques années. La critique du concept de «Nouvelle Hanse » permit aux nordiques de rappeler leurs spécificités. Ces ambitions furent très vite déçues notamment face à la formulation d'une stratégie intégrée pour la mer Baltique dès 2006 et cela bien que celle-ci travaille en collaboration avec la « dimension septentrionale». Le gouvernement suédois a fait de cette stratégie macrorégionale baltique, qui exclut directement la Russie, une priorité pour sa présidence de l'Union Européenne en 2009 avant de s'y désintéresser. La Baltique reste donc une région sans centre et la relance apparait dès lors délicate.

Est-ce l'occasion, une nouvelle fois, de réveiller la métaphore hanséatique délaissée au milieu des années 1990 ? La vision de Björn Engholm, qui n'a pas vraiment pu lui survivre, a-t-elle une chance de revoir le jour? La métaphore de la Hanse, récur-

${ }^{28}$ Ibid., 152. 
rente dans l'esprit des penseurs baltiques, n'aurait-elle pas un rôle de relance dans des moments où la coopération s'épuise... La Hanse serait-elle la régulation presque biologique donnant l'impulsion à « des cycles d'unification baltique »? Tout comme la construction européenne, la construction de l'unité baltique, plus politique que naturelle, davantage créée que remémorée, n'est pas une ligne continue mais s'avère cyclique, connaissant flux et reflux.

En se débarrassant du mot «Hanse » trop polémique, la coopération baltique doit être perçue davantage comme un ensemble de cercles (grappes des coopérations nordiques, baltiques, baltes, bilatérales, de Barents, européennes) se recoupant ou non que comme une région à proprement parler uniforme et bornée. Il faut rappeler la forme réticulaire des coopérations baltiques qui fait la force de la région. En dehors de l'Europe, la Baltique fonctionnelle intègre la Russie ou la Biélorussie. "L'unité d'une région baltique » est un concept trop simple. La Baltique mérite d'être pensée dans la complexité : elle offre l'occasion d'un emboitement des aires de coopérations se recoupant en graphes au gré des rayons convergents.

Nicolas Escach 